\title{
Detection of Sparse Damages in Structures
}

\author{
Natalia Sabourova, Niklas Grip, Ulf Ohlsson, Lennart Elfgren \\ Lulea University of Technology, SE-971 87 Lulea, Sweden, \\ Yongming Tu \\ School of Civil Engineering, Southeast University, Nanjing, China, \\ Ivan Duvnjak, Domagoj Damjanović \\ University of Zagreb, Croatia
}

Contacting author: natalia.sabourova@Itu.se, lennart.elfgren@Itu.se

\begin{abstract}
Structural damage is often a spatially sparse phenomenon, i.e. it occurs only in a small part of the structure. This property of damage has not been utilized in the field of structural damage identification until quite recently, when the sparsity-based regularization developed in compressed sensing problems found its application in this field.

In this paper we consider classical sensitivity-based finite element model updating combined with a regularization technique appropriate for the expected type of sparse damage. Traditionally, (I), $l_{2}$ norm regularization was used to solve the ill-posed inverse problems, such as damage identification. However, using already well established, (II), $l_{1}$-norm regularization or our proposed, (III), $l_{1}$-norm total variation regularization and, (IV), general dictionary-based regularization allows us to find damages with special spatial properties quite precisely using much fewer measurement locations than the number of possibly damaged elements of the structure. The validity of the proposed methods is demonstrated using simulations on a Kirchhoff plate model. The pros and cons of these methods are discussed.
\end{abstract}

Keywords: sparse damage; $l_{2}$-norm; $l_{1}$-norm; total variation; dictionary-based regularization, sensitivity

\section{Introduction}

This paper is devoted to identification of welllocalized, or in other words, spatially sparse structural damages. In reinforced concrete structures, spatially sparse damage patterns can be associated with, for example, shear cracks. When such cracks develop, a brittle failure of the structure may be close - an inclined crack can find its way through a structure, without being prevented by reinforcement. It is of great interest to identify location and severity of such local cracks more precisely without smoothing the damage to the areas nearby and in this way to distinguish these cracks from other less severe cracks, such as e.g. bending cracks.

The damage identification methods presented here are based on the classical iterative sensitivitybased finite element model updating (FEMU) method combined with regularization techniques which help to detect damages with the expected properties [1-7].

In sensitivity-based FEMU approach, damage detection is considered as an inverse problem, i.e. 
knowing the model outputs, one needs to obtain the internal model parameters. In a damage identification content, these model parameters, usually the elasticity modulus for an element or for a group of elements, are related to so-called damage parameters. The updating of damage parameters and thus the finite element model is driven by minimization of the difference or socalled residual between the model and the corresponding measured properties for the damaged and the undamaged structure. Such properties can be, for example, dynamic properties, such as natural frequencies and the corresponding mode shapes of vibration. To make use of very efficient computational algorithms, the residual is quite often linearized, and the damage detection problem is transformed to the solution of a system of linear equations $[5,6,8]$.

There are two main problems connected to the described damage identification approach. First, in the presence of noise in the outputs, which is the case with vibration tests, the inverse problem becomes ill-posed, i.e. small variations in the outputs lead to unreasonably large variations in the damage and thus the model parameters. Second, the linear system associated with the damage identification problem is usually underdetermined and therefore has an infinite number of solutions for damage parameters. Both these problems can be solved by using regularization methods, which are increasingly more often considered in structural damage identification.

In this paper, we investigate different regularization methods for the damage identification problem starting from (I) classical Tikhonoff or $l_{2}$-norm regularization, (II) sparse $l_{1^{-}}$ norm regularization, (III) $l_{1}$-norm total variation regularization and (IV) dictionary-based regularization. We compare these regularization methods when using them for damage identification on a simulated Kirchhoff plate with well-localized damage. The simplicity of the studied simulation model should not be underestimated, since, a number of real structures can be considered as examples of "Kirchhoff plates" (see Figure 1).

In the accompanying paper [9] we compared the damage localization possibilities of the here presented sensitivity-based model updating method regularized by $l_{1}$-norm with a simple model-free method, named Mode Shape Damage Index, which is based only on comparison of the undamaged and damaged normalised mode shape vectors.

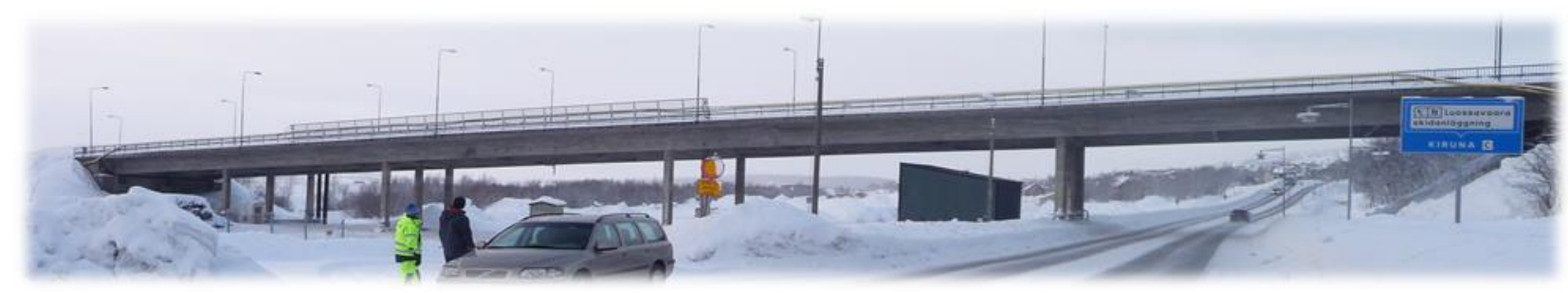

Figure 1. The Mine bridge in Kiruna in northern Sweden is an example of a structure where sparsitybased methods to detect possible defects in the bridge slab would be valuable [10].

\section{Damage detection as a minimization problem}

\subsection{Damage parametrization}

It is common in the literature on structural damage detection to parameterize the stiffness matrix as follows $[1,5-7,11]$

$$
K(\alpha)=K^{0}-\sum_{i=1}^{n} \alpha_{i} K_{i}
$$

where $K(\alpha)$ is the improved stiffness matrix of the parameterized model; $K_{i}$ is the constant stiffness matrices for the $i^{\text {th }}$ single element or group of elements representing the uncertain model property and location; $K^{0}$ is the matrix corresponding to the initial undamaged structure; 
and $n$ is the number of independent model parameters.

The dimensionless updating parameter $\alpha_{i}$, also called damage index, corresponds to the local reduction of the elasticity modulus [12], thereby inducing the reduction of bending stiffness derived in elastic beam or plate theory, namely

$$
\alpha_{i}=\frac{E_{i}^{0}-E_{i}}{E_{i}^{0}}
$$

where $E_{i}^{0}$ and $E_{i}$ are the undamaged and damaged elasticity modulus of the $i^{\text {th }}$ single element or group of elements.

A good damage detection method should provide small $\alpha_{i}$, or zero in the ideal case, indicating the absence of damage and positive $\alpha_{i}$ indicating the decrease in the elasticity modulus and therefore indicating damage for a single element or for a group of elements.

\subsection{Problem formulation}

The estimation of the damage indices $\alpha_{i}$ is performed by minimizing the residual or difference between the damaged and undamaged dynamic properties. For example, one defines

$$
\begin{gathered}
r_{\lambda_{j}}(\alpha)=w_{\lambda_{j}}\left(1-\frac{\lambda_{j}(\alpha)}{\lambda_{j}^{d}}\right) \\
r_{\phi_{j}}(\alpha)=w_{\phi_{j}}\left(\frac{\left(\phi_{j}^{d}\right)^{T} \phi_{j}(\alpha)}{\left\|\phi_{j}(\alpha)\right\|_{2}^{2}} \phi_{j}^{d}\right. \\
\left.-\phi_{j}(\alpha)\right)
\end{gathered}
$$

where $r_{\lambda_{j}}$ is the $j^{\text {th }}$ component of the eigenvalue residual and $r_{\phi_{j}}$ is the residual vector for the $\phi_{j}$ mode shape, $w_{\lambda_{j}}$ and $w_{\phi_{j}}$ are the corresponding weights. The upper index $d$ is referring to the damaged property, a property without upper index is undamaged. Then, the damage detection problem, in its simple formulation, is about minimization

$$
\min _{0 \leq \alpha_{i} \leq 1} \frac{1}{2}\|r(\alpha)\|_{2}^{2}
$$

The residual (3) corresponding to small changes in the dynamic properties of the structure can be linearly related to small changes $\Delta \alpha$ in the damage indices around $\alpha$ as follows

$$
r=S \Delta \alpha+\varepsilon
$$

Here the so-called sensitivity matrix $S \in R^{m \times n}$ is defined by

$$
S_{i j}=\frac{\partial r_{i}}{\partial \alpha_{j}}
$$

Typically, the number of residuals $m$ is smaller than the number of damage indices, i.e. $m<n$ and system (5) is underdetermined. The error vector $\boldsymbol{\varepsilon}$ is introduced to represent this effect as well as the effect of the measurement noise and numerical errors. The derivatives in (6) are usually found by either Fox-Kapoor [13] or Nelson [14, 15] methods, depending on if a structure in question has repeated (associated with symmetrical structures) or non-repeated eigenvalues. Then, using (5) one usually solves the following problem

$$
\min _{0 \leq \alpha_{i} \leq 1} \frac{1}{2}\|S \Delta \alpha-r(\alpha)\|_{2}^{2}
$$

\subsection{Problem regularization}

To address the problems related to infinite number of solutions of underdetermined system, measurement noise and numerical errors and thus to solve the problem (5), different regularization techniques can be used. The expected damage pattern defines the optimal regularization.

\section{$2.4 l_{2}$-norm regularization}

Tikhonov or $l_{2}$-norm regularization belongs to the traditional and most used regularization technique $[1,5]$. The problem (7) is then formulated as follows

$$
\min _{0 \leq \alpha_{i} \leq 1} \frac{1}{2}\|S \Delta \alpha-r(\alpha)\|_{2}^{2}+\rho\|\Delta \alpha\|_{2}^{2}
$$


where here in what it follows $\rho>0$ is a regularization coefficient. However, in the case of detection of the spatially sparse damage, this regularization significantly smooths the solution vector $\Delta \alpha$. See Figures 2, 4, 5 b.

\section{$2.5 l_{1}$-norm regularization}

Only quite recently $l_{\mathbf{1}}$-norm regularization was recognized in the field of structural damage detection $[6-8,16-18]$. The reason is that it provides the most efficient way to find a sparse solution of system (5) [2]. The problem (7) is then

$$
\min _{0 \leq \alpha_{i} \leq 1} \frac{1}{2}\|S \Delta \alpha-r(\alpha)\|_{2}^{2}+\rho\|\Delta \alpha\|_{1}
$$

where $\rho>0$ is a regularization coefficient. The $l_{\mathbf{1}}$ norm belongs to a class of convex regularizers and thus making the problem (9) convex. For convex problems there exist very efficient open source solvers.

\section{6 $l_{1}$-norm total variation regularization}

Assume that the vector $\alpha$ of damage indices is distributed over a 2D grid as follows

$$
A=\left[\begin{array}{cccc}
\alpha_{1} & \alpha_{d_{1}+1} & \ldots & \alpha_{\left(d_{2}-1\right) d_{1}+1} \\
\alpha_{2} & \alpha_{d_{1}+2} & \ldots & \alpha_{\left(d_{2}-1\right) d_{1}+2} \\
\vdots & \vdots & \ddots & \vdots \\
\alpha_{d_{1}} & \alpha_{2 d_{1}} & \ldots & \alpha_{d_{1} d_{2}}
\end{array}\right]
$$

with $d_{1} d_{2}=n$, where $n$ is the total number of damage indices. For example, for a rectangular $r \times c$ plate structure with $c$ elements in one row and $r$ elements in one column for which a damage index is defined for each element we have $d_{1}=r$ and $d_{2}=c$. Denote the element of this matrix at row $i$ and column $j$ by $A_{i j}$ and define the operators

$$
\begin{aligned}
& D_{h_{i}, j A}=\left\{\begin{array}{c}
A_{i+1, j}-A_{i j}, \\
0,
\end{array}\right. \\
& D_{v_{i}, j A}=\left\{\begin{array}{c}
A_{i, j+1}-A_{i j}, \\
0,
\end{array}\right.
\end{aligned}
$$

where $D_{h}$ stands for differences between horizontal rows and $D_{v}$ stands for differences between vertical columns of the matrix $A$.
Compose a "discrete gradient" of $A$ at row $i$ and column $j$ by $D_{i, j A}=\left[\begin{array}{l}D_{h_{i}, j A} \\ D_{v_{i}, j A}\end{array}\right]$ and define its length

$$
d_{i, j A}=\left\|D_{i, j A}\right\|_{2}=\sqrt{D_{h_{i}, j A}^{2}+D_{v_{i}, j A}^{2}}
$$

Then the isotropic (invariant under rotation) total variation of $A$ is given by

$$
\operatorname{Var}_{1}(A)=\sum_{i, j} d_{i, j A}
$$

Now we consider the following minimization problem [7]

$$
\min _{0 \leq \alpha_{i} \leq 1} \frac{1}{2}\|S \Delta \alpha-r(\alpha)\|_{2}^{2}+\rho \operatorname{Var}_{1}(\Delta \alpha)
$$

The total variation, being a smoothing term, together with the sparsifying $\boldsymbol{l}_{\mathbf{1}}$-norm implies sparsity of damage indices differences and thus resulting in a piecewise constant damage index vector with sharp edges.

\subsection{Dictionary regularization, containing, for instance, a wavelet basis}

Assume that a sparse damage index vector $\alpha$ can be decomposed into a series expansion

$$
\alpha=\sum_{n} c_{n} \psi_{n}
$$

with only a few negligible coefficients $c_{n}$. Let us define a dictionary of vectors $\psi_{n}$ stored as column vectors in a matrix $\Psi$. For example, we can let $\psi$ be the $n \times n$ identity matrix, where $n$ is the length of the damage index vector $\alpha$, if we are only looking for point damage(s), or we can try with a wavelet basis for other type of sparse damages, say damage cracks. For detection of both point damages and cracks, we can combine these two dictionaries into a twice as large $n \times 2 n$ dictionary $\Psi$. This gives an analysis mapping

$$
\alpha \mapsto c=\Psi^{*} \alpha
$$

for computing the series expansion coefficient. Here we write $M^{*}$ for the complex conjugate of matrix $M$. For the corresponding reconstruction or synthesis of $\alpha$ from the coefficients $c$, we can use the theory of frames and Riesz bases described in more detail, for example, in [20-22]. In general, the 
matrix $\Psi$ is not invertible or even square, but its columns can be described as a frame for their span with frame bounds closely related to the condition number of $\psi$. Associated with $\psi$ is the corresponding canonical dual frame

$$
\widetilde{\Psi}=\left(\Psi \Psi^{*}\right)^{-1} \Psi
$$

that gives the synthesis mapping

$$
\alpha \mapsto c=\widetilde{\Psi}_{c} \stackrel{\text { def }}{=} \sum_{n} c_{n} \tilde{\psi}_{n}
$$

Let $c=\Psi^{*} \alpha$ be the wavelet coefficients corresponding to the damage index vector $\alpha$. Then, $\alpha=\widetilde{\Psi_{\mathrm{C}}}$ and we formulate the minimization problem

$$
\min _{0 \leq \alpha_{i} \leq 1} \frac{1}{2}\|S \Delta \alpha-r(\alpha)\|_{2}^{2}+\rho\|c\|_{1}
$$

For the special case of a square matrix $\Psi$ with orthonormal column vectors, we get $\widetilde{\Psi} \widetilde{\Psi}=\Psi$.

Other approaches in this area are presented in [23] and [24].

\section{Damage detection on a Kirchhoff plate}

We test our methods on a Kirchhoff rectangular plate with size $1 \mathrm{~m} \times 1.05 \mathrm{~m} \times 0.01 \mathrm{~m}$. The model is built using four-node-shell elements with each node having six degrees of freedom: three translational and three rotational. The size of each finite element is $0.05 \mathrm{~m} \times 0.05 \mathrm{~m}$. Thus, the model contains 420 elements. The plate is fixed on all its sides.

In all simulations the noise is modelled by

$$
\hat{\psi}=\psi(1+\varepsilon \sigma)
$$

where $\hat{\psi}$ is the estimated vibration parameter, $\psi$ is the corresponding exact parameter, $\sigma$ is a normally distributed random variable with zero mean and a variance of 1.0 , the variation $\varepsilon$ is equal to 0.001 . The regularization methods are implemented by using open-source CVX Matlab software [19].

Damage is simulated by reducing the elasticity modulus for the chosen element or group of elements.

\subsection{Simulation results}

Figures 2-5 show the identification results for a point, a two-point, a line and a rectangular type of damage scenarios.

As expected, $l_{2}$-norm regularization (I) smooths out the effect of damage to the surrounding elements and thus cannot be the best choice for identification of spatially sparse damages or damages with sharp edges, see Figures 2, 4, $5 \mathrm{~b}$. On the other hand, $l_{1}$-norm (II) is very effective in identification of point damage(s), see Figures $2 \mathrm{c}-\mathrm{d}$, $3 \mathrm{c}$. When the damage pattern is spread out over a region and has sharp edges to the undamaged area then the most suitable identification is via $l_{\mathbf{1}}$-norm total variation regularization (III), see Figure $5 \mathrm{~d}$. Damages of line type can be identified, by using, for example, dictionary-based regularization (IV), see Figure $4 \mathrm{~d}$. Regularization based on specially targeted dictionary can be used in identification of all these types of damages.

In an accompanying paper [9] we compared the sensitivity-based model updating combined with $l_{1}$ norm regularizer (II) with a computationally much more simple damage detection method, a kind of model assurance criterion method, a so-called Mode Shape Damage Index (MSDI). For a simple simulation setup, the damage localization property of the two methods gave comparable results. However, as expected, the MSDI results were more blurry. It would be interesting to investigate if sparsity could also be introduced into such simple methods.

\section{Conclusions}

In this paper we investigated a sensitivity-based FEMU method combined with several regularization techniques to identify damages with special properties of spatial sparsity.

It has been recognized quite recently that traditional sensitivity-based FEMU supplied with $l_{2}$-norm regularization (I) is not capable to precisely identify sparse damages or damages with sharp edges, as it was also shown in this paper. On the other hand, sparsity-based solutions, such as $l_{\mathbf{1}^{-}}$ norm (II), $l_{1}$-norm total variation (III) and 
dictionary-based regularizations (IV) can be successfully used in detection of damages with special spatial properties using, in addition, fewer spectrum components than the number of potentially damaged elements of the structure.

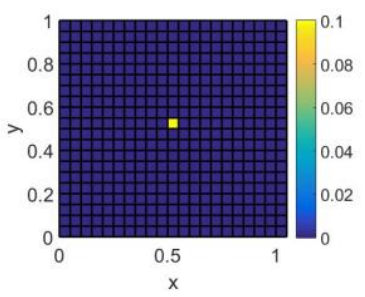

a)

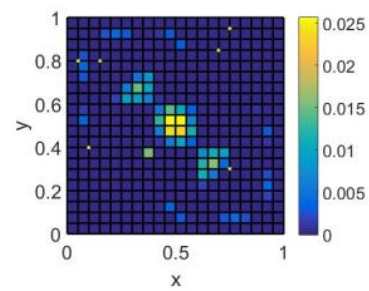

b)

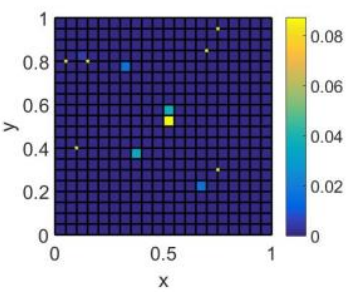

c)

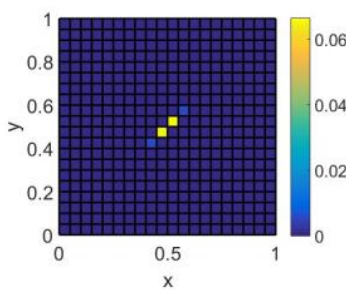

d)

Figure 2. Point damage and damage identification results. a) True damage, b) Damage pattern found by $l_{2}$-norm regularization (I) based on the first 8 natural frequencies and mode shapes; c) Damage $\approx 8.71 \%$ found by $l_{1}$-norm regularization (II) based on the first 8 natural frequencies and mode shapes, d) Damage $\approx 6.63 \%$ found by $l_{1}$-norm regularization (II) based on the first 10 natural frequencies. Measurement locations in all simulations are denoted by yellow stars.

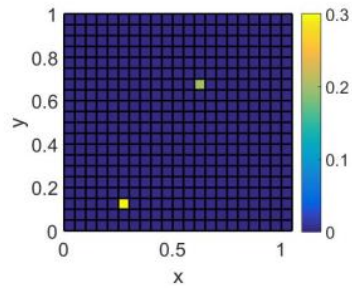

a)

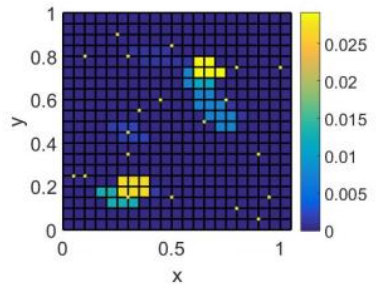

b)

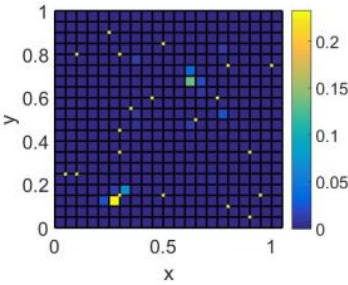

c)

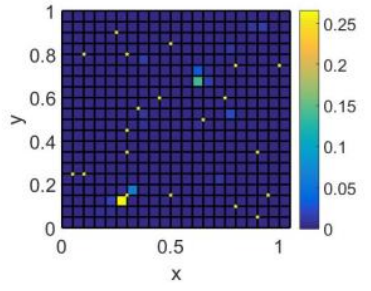

d)

Figure 3. Two-point damage and damage identification results. a) True damage, b) Damage pattern found by $l_{1}$-norm total variation (III) regularization based on the first 15 natural frequencies and mode shapes; c) Damage $\approx 30.95 \%$ and $\approx 19.63 \%$ found by $l_{1}$-norm regularization (II) based on the first 10 mode shapes; d) Damage $\approx 26.52 \%$ and $\approx 13.80 \%$ found by dictionary-based regularization (IV) based on the first 15 natural frequencies and mode shapes. All damage identification results are based on 20 measurement locations denoted by yellow stars.

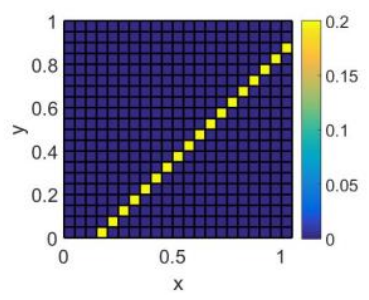

a)

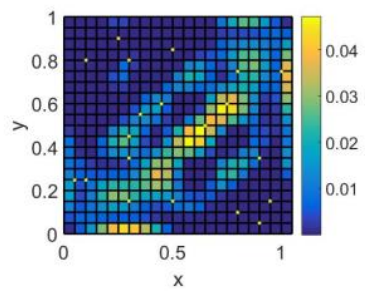

b)

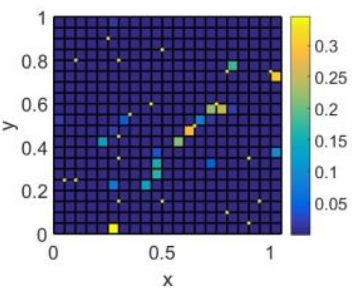

c)

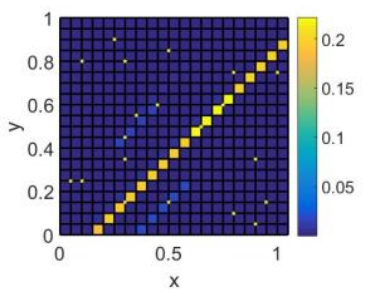

d)

Figure 4. Line damage and damage identification results. a) True damage, b) Damage pattern found by $l_{2}$ norm regularization (I); c) Damage pattern found by $l_{1}$-norm regularization (II); d) Damage pattern found by dictionary-based regularization (IV). All damage identification results are based on first 10 natural frequencies and mode shapes and 20 measurement locations denoted by yellow stars. 


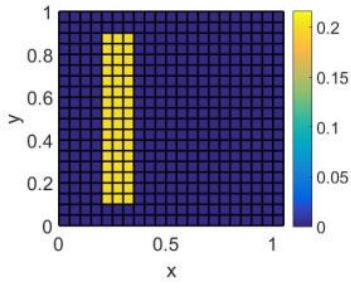

a)

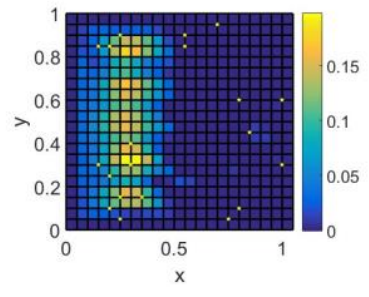

b)

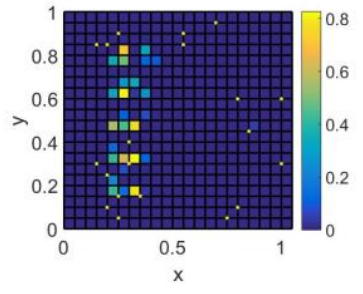

c)

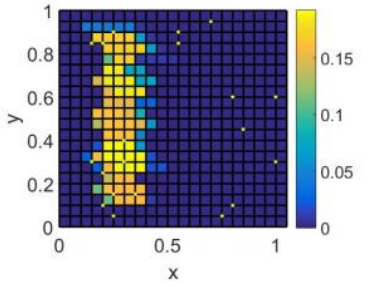

d)

Figure 5. Rectangular damage and damage identification results. a) True damage, b) Damage pattern found by $l_{2}$-norm regularization (I); c) Damage pattern found by $l_{1}$-norm regularization (II); d) Damage found by $l_{1}$-norm total variation regularization (III). All damage identification results are based on the first 17 natural frequencies and mode shapes and 20 measurement locations denoted by yellow stars.

\section{References}

[1] Friswell M.I. and Mottershead J.E. Finite Element Model Updating in Structural Dynamics. Dordbrecht: Kluwer Academic, 1995.

[2] Bruckstein A., Donoho D. and Elad M. From Sparse Solutions of Systems of Equations to Sparse Modelling of Signals and Images. SIAM Review. 2009; 51(1): 34-81.

[3] Elad M. Sparse and Redundant Representations. From Theory to Applications in Signal and Image Processing. New York: Springer, 2010.

[4] Boyd S. and Vandenberghe L. Convex Optimization. Basel: Cambridge University Press New York, Boston, 2004.

[5] Weber B., Paultre P. and Proulx J. Consistent Regularization of Nonlinear Model Updating for Damage Identification. Mechanical Systems and Signal Processing. 2008; 23(6): 1965-1985.

[6] Hernandez E.M. Identification of Isolated Structural Damage from Incomplete Spectrum Information Using I1-norm Minimization. Mechanical System and Signal Processing. May 2014; 46(1): 59-69.

[7] Grip N., Sabourova N. and Tu Y. Sensitivitybased Model Updating for Structural Damage Identification Using Total Variation Regularization.
Mechanical systems and signal processing. 2017; 84(A): 365-383.

[8] Fritzen C.-P. and Ginsberg D. Sparse Solution techniques in Load and Damage Monitoring Systems. Advances in Science and Technology. 2017; 101: 35-44.

[9] Duvnjak I., Damjanović D., Sabourova N., Grip N., Ohlsson U., Elfgren L. and Tu Y. Damage Detection in Structures - Examples. IABSE Symposium 2019, March 27-29, Guimarães, Portugal, 2019.

[10] Huang Z., Tu. Y., Grip N., Sabourova N., Bagge N., Ohlsson U. and Elfgren L. Modelling of Damage and Its Use in Assessment of a Prestressed Concrete Bridge. 19th IABSE Congress, Stockholm September 2016, 16 pp.

[11] Link M. Updating of Analytical Models Review of Numerical Procedures and Application Aspects. In "Structural Dynamics @ 2000: current status and future directions", 2001, 193-223.

[12] Lemaitre J. and Desmorat R. Engineering damage mechanics. Ductile, Creep, Fatigue and Brittle Failures. Berlin: Springer; 2005.

[13] Fox R.L. and Kapoor M.P. Rate of change of eigenvalues and eigenvectors, AIAA Journal. 1998; 6(12): 2426-2429. 
[14] Nelson R.B. Simplified Calculation of Eigenvector Derivatives. AIAA Journal. 1976; 14(9): 1201-1205.

[15] Friswell M.I. The Derivatives of Repeated Eigenvalues and Their Associated Eigenvectors. Journal of Vibration and Acoustics. 1998; 118(3): 390-397.

[16] Cortial J., Farhat C., Guibas L. J. and Rajashekhar M. Compressed Sensing and TimeParallel Reduced-Order Modeling for Structural Health Monitoring Using a DDDAS. Lecture Notes in Computer Science. 2007; 4487: 1171-1179.

[17] Yuequan B., Shumei Z., Hui L., and Jinping $O$. Structural damage identification based on substructure sensitivity and sparse regularization. In Proceedings of SPIE - The International Society for Optical Engineering, April 2013.

[18] Zhang C.D. and Xu Y. L. Comparative Studies on Damage Identification with Tikhonov Regularization and Sparse Regularization. Structural Control and Health Monitoring. March 2015; 23(3): 560-579.

[19] CVX: Matlab Software for Disciplined Convex Programming. Online software and documentation. http://cvxr.com/cvx.

[20] Gröchenig K. Foundations of Time-Frequency Analysis. Birkhäuser, 2000.

[21] Christensen O. An Introduction to Frames and Riesz Bases. Birkhäuser, 2002.

[22] Grip N. Wavelet and Gabor Frames and Bases: Approximation, Sampling and Applications. Doctoral thesis 2002:49, Luleå University of Technology.

[23] Nagarajaiah S. \& Yang Y. Modeling and harnessing sparse and low-rank data structure: a new paradigm for structural dynamics, identification, damage detection, and health monitoring. Structural Control and Health monitoring, 2016; 24: e1851, 22 pp. DOI: 10.1002/stc.1851.
[24] Sen D., Aghazadeh A., Mousavi A., Nagarajaiah S. \& Baraniuk R. Sparsity-based approaches for damage detection in plates. Mechanical Systems and Signal Processing, vol 117 (2019), p 333-346. 